\title{
The Epidemiology of Posttraumatic Epilepsy
}

\author{
Jakob Christensen, MD, PhD ${ }^{1}$ \\ 1 Department of Neurology, Aarhus University Hospital, \\ Aarhus, Denmark
}

Address for correspondence Jakob Christensen, MD, PhD, Department of Neurology, Aarhus University Hospital, Norrebrogade

Semin Neurol 2015;35:218-222.

\begin{abstract}
Keywords

- traumatic brain injury

- seizures

- epilepsy

- posttraumatic

- epidemiology

Traumatic brain injury is an important contributor to morbidity and mortality, and results in reduced quality of life and lifespan: An estimated 1.7 million traumatic brain injuries occur annually in the United States alone. Traumatic brain injury carries an increased risk of epilepsy that correlates with the severity of the brain injury. Posttraumatic epilepsy accounts for less than 10\% of epilepsy, but traumatic brain injury is one of only a few potentially preventable causes of epilepsy. Despite several well-controlled human studies, there is no current preventive treatment available for humans. Therefore, primary prevention is the only proven way to prevent posttraumatic epilepsy.
\end{abstract}

\section{Epidemiology of Traumatic Brain Injury}

Traumatic brain injury (TBI) is an important contributor to morbidity and mortality, and results in reduced quality of life and lifespan. ${ }^{1}$ An estimated 1.7 million traumatic brain injuries occur annually in the United States ${ }^{2} ; 1.2$ million traumatic brain injuries occur annually in the European Union (including Iceland, Norway, and Switzerland). ${ }^{3}$ The risk of TBI varies greatly with age, being particularly high in early childhood and in the elderly, but the incidence also has a striking peak incidence in early adulthood-especially among males (-Fig. 1). Traumatic brain injury is more frequent in males than in females; it is overall approximately1.4 times more common among males (-Fig. 1). ${ }^{2}$

The consequences of TBI are significant, not least in terms of increase in mortality. Mortality following TBI remains low until 15 years of age, but from that age onward the mortality increases sharply (-Fig. 2). There are marked sex differences, with mortality from TBI being 2.9 times more likely in males than in females (-Fig. 2). ${ }^{2}$ Approximately 50,000 persons in the United States die annually from TBI-related injuries. Traumatic brain injury results from several causes, including falls and motor vehicle accidents ( - Fig. 3 ). The causes of injury relate closely to age-for example, TBI from motor vehicle accidents and assaults peak in adolescence and early adulthood, coinciding with the sharp rise in incidence and mortality (for males) associated with TBI in that age group (-Fig. 3). ${ }^{2}$
It is extremely difficult to estimate the costs of TBI and more difficult to compare these estimates across countries or even continents. However, it has been estimated that the total costs of TBI in the European Union (plus Iceland, Norway, and Switzerland) with a total population of 514 million, is more than 33 billion euros (equivalent to 37 billion US\$ or 24 billion GBPE) this compares to the total cost of stroke of 64 billion euros (72 billion US\$ or 47 billion GBP£ per year). ${ }^{3}$ It is currently unknown what additional part comorbid epilepsy plays in the economic cost of the consequences of TBI.

\section{Epidemiology of Seizures and Epilepsy after Traumatic Brain Injury}

Seizures occurring early after TBI are usually distinguished from epilepsy (recurrent unprovoked seizures) because they differ in mortality and prognosis. ${ }^{4}$ The following definitions have been proposed: (1) immediate seizures, which occur less than 24 hours after injury; (2) early seizures, which occur less than 1 week after injury; and (3) late seizures, which occur more than a week after injury and constitute the diagnosis of posttraumatic epilepsy. ${ }^{5-7}$

\section{The Epidemiology of Immediate and Early Posttraumatic Seizures}

The risk of early seizures ranges from approximately $2 \%^{8,9}$ in population-based studies to 14 to $30 \%$ in patients with severe

Copyright $\odot 2015$ by Thieme Medical Publishers, Inc., 333 Seventh Avenue, New York, NY 10001, USA. Tel: +1(212) 584-4662.
DOI http://dx.doi.org/ $10.1055 / \mathrm{s}-0035-1552923$ ISSN 0271-8235. 
Estimated Average Annual Rates of Traumatic Brain Injury by Sex, United States, 2002-2006

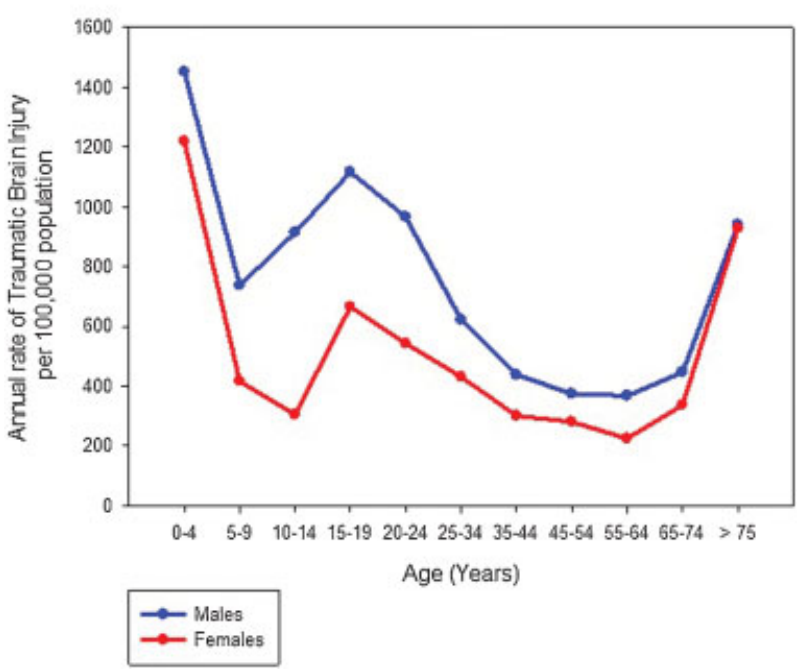

Fig. 1 Annual rate of traumatic brain injury by sex in the United States, 2002-2006. ${ }^{2}$

TBI. ${ }^{10}$ Temkin reviewed the risk factors for early seizures and reported that depressed skull fracture, intracerebral hematoma, and subdural hematoma were associated with about a $25 \%$ risk of immediate or early posttraumatic seizures. ${ }^{10}$ Immediate seizures and early seizures are also risk factors developing later epilepsy ${ }^{10}$ and seizures within the first 24 hours have even been used to identify patients with a high risk of epilepsy after TBI (and thus being eligible for studies of posttraumatic epilepsy). ${ }^{9}$ However, immediate and early posttraumatic seizures are also markers of severity of the brain injury and thus may explain the association observed with the development of late seizures (epilepsy).

Rates of Traumatic Brain Injury-Related Deaths, by Age Group and Sex United States, 2002-2006

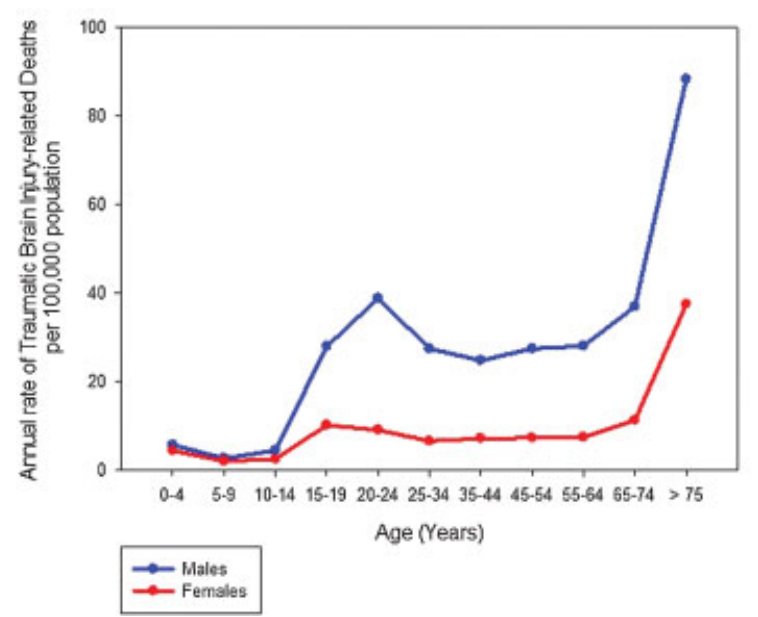

Fig. 2 Rates of traumatic brain injury-related deaths by age group and sex in the United States, 2002-2006. ${ }^{2}$

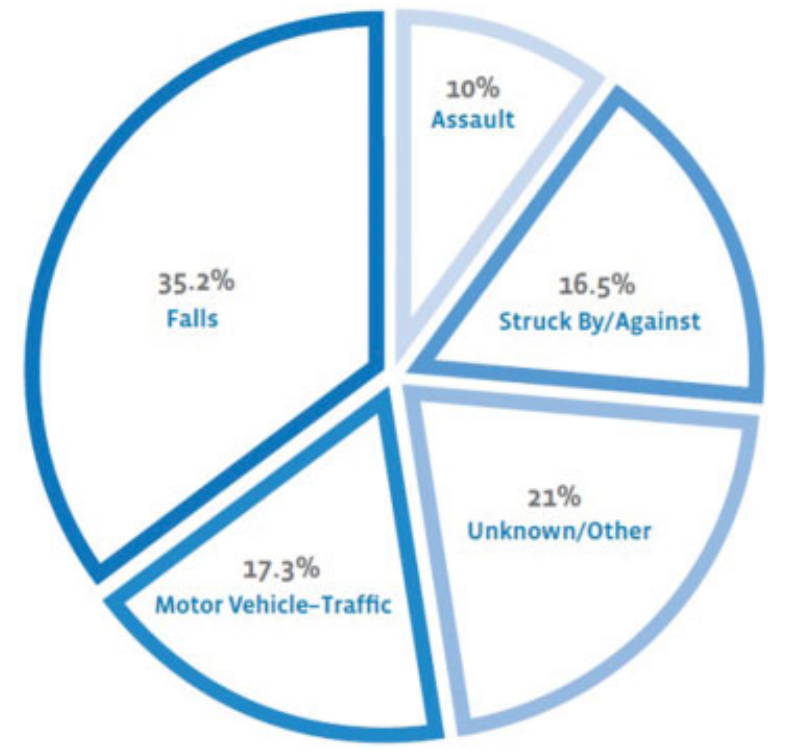

Fig. 3 Traumatic brain injury by external cause in the United States, 2002-2006. ${ }^{2}$

\section{Population based Epidemiology of Epilepsy after Traumatic Brain Injury}

With the new definition of epilepsy proposed by the International League Against Epilepsy (ILAE) and the International Bureau for Epilepsy (IBE), epilepsy is a disorder of the brain characterized by an enduring predisposition to generate epileptic seizures. ${ }^{11,12}$ The definition of epilepsy requires the occurrence of at least one epileptic seizure. ${ }^{12}$ Thus, a single, late, unprovoked seizure in a person with a structural TBI fulfills the diagnostic criteria for epilepsy. In accordance with this, the risk of recurrent late seizures 2 years after TBI was $86 \%$ in a study of 63 moderately-to-severely head-injured adults who developed late posttraumatic seizures. ${ }^{13}$

The most important and constant finding when studying posttraumatic epilepsy is that the severity of brain injury is associated with the risk of subsequent epilepsy. ${ }^{8,14}$ In a follow-up study from Denmark, the authors followed more than 1.6 million children and young adults for up to 30 years after TBI. Relative to no brain injury, the relative risk (RR) of epilepsy was twice as high after mild TBI $(\mathrm{RR}=2.22,95 \%$ confidence interval [CI]: 2.07-2.38); 7 times higher after severe brain injury ( $\mathrm{RR}=7.40,95 \% \mathrm{CI}$ : 6.16-8.89); and twice as high after skull fracture $(\mathrm{RR}=2.17,95 \% \mathrm{CI}: 1.73-2.71) .{ }^{14}$ The risk of epilepsy was highest shortly after the TBI, but remained increased for more than 10 years after TBI ( - Fig. 4). The risk was highest in persons older than 15 years of age at the time of TBI. The relative risk of posttraumatic epilepsy was higher in women than in men, and the risk was higher in people with a family history of epilepsy. ${ }^{9}$

A study from Olmsted County, Minnesota, identified 4,541 children and adults with TBI occurring between 1935 and $1984 .^{10}$ The standardized incidence ratio for epilepsy compared with the background population was 1.5 (95\% CI: 1.0-2.2) after mild TBI, 2.9 (95\% CI: 1.9-4.1) after moderate 


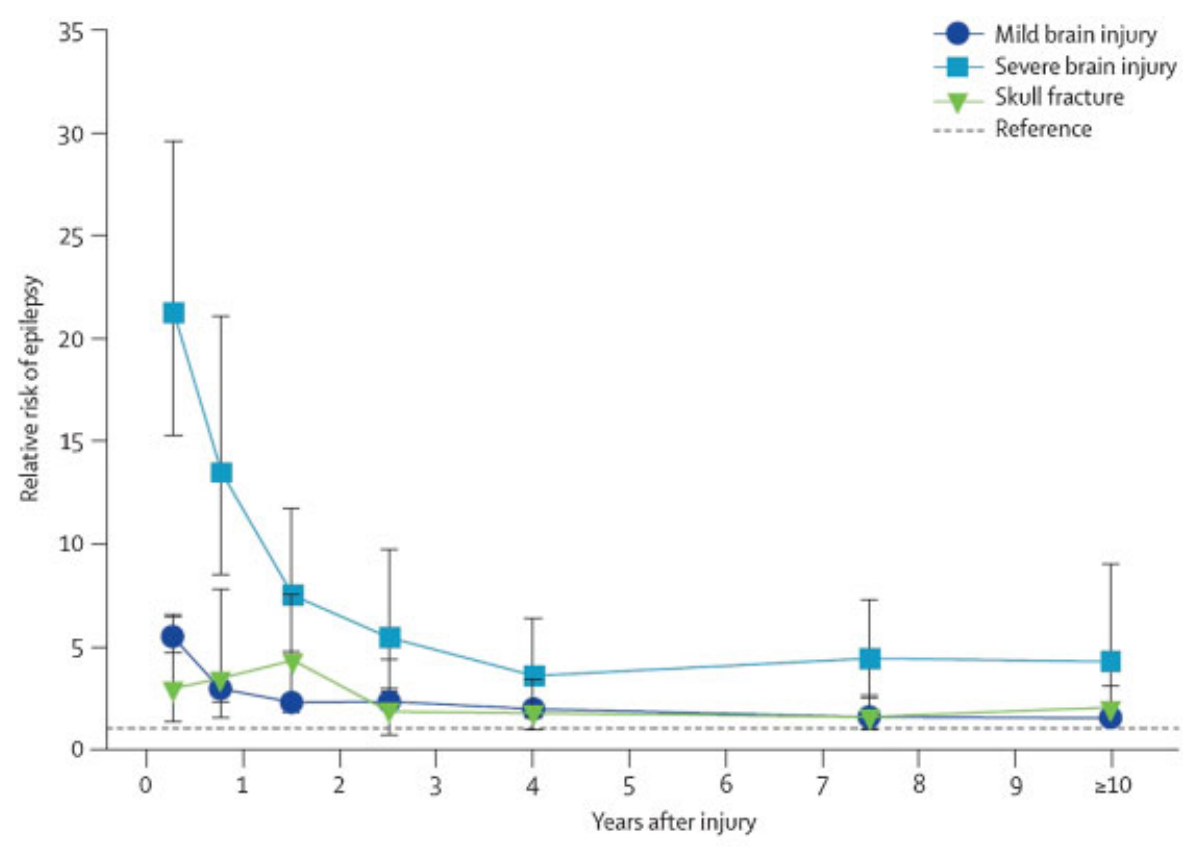

Figure: Relative risk of epilepsy after brain injury in Denmark (1977-2002)

Fig. 4 Relative risk of epilepsy after brain injury in Denmark, 1977-2002. ${ }^{14}$

TBI, and 17.0 (95\% CI: 12.3-23.6) after severe TBI. $^{15}$ The authors identified brain contusion with subdural hematoma, skull fracture, loss of consciousness or amnesia for more than one day, and an age of 65 years or older as risk factors for epilepsy. ${ }^{8}$

A population-based U.S. study followed 2,118 persons hospitalized with TBI for 3 years and determined the cumulative incidence of posttraumatic epilepsy. ${ }^{15}$ After adjusting for the significant loss to follow-up (55\% at 3 years), they found a risk of 4.4 per 100 persons for mild TBI, 7.6 per 100 persons for moderate TBI, and 13.6 per 100 persons for severe TBI. A history of psychiatric depression increased the risk of posttraumatic epilepsy. ${ }^{15}$ A study from Sweden found that in children with TBI, eight (7\%) of 109 developed immediate seizures and 12 (11\%) developed epilepsy. Of these 12, 5 had had immediate seizures. ${ }^{16}$

\section{Estimating the Absolute Risk of Epilepsy after Traumatic Brain Injury}

There are significant risks of epilepsy following TBI, especially following severe TBI, ${ }^{8,14}$ and even mild TBI (concussion) seems to carry an increased long-term relative risk of epilepsy. This relative risk may remain increased more than 10 years after injury. ${ }^{14}$ Therefore, it is important to be aware of symptoms that may represent epileptic seizures in persons with TBI. Pilots and truck drivers have occupations where there is a special need to be aware of the risk of epilepsy following TBI. ${ }^{17}$ Even though the relative risk of epilepsy after brain injury may be increased compared with the population without, it is important to estimate the absolute risk of posttraumatic epilepsy among these patients. $^{8,14}$
Clinical case series and population-based studies estimate cumulative incidence of epilepsy after TBI. The probability of developing late posttraumatic seizures in the entire sample of 480 persons with TBI in the United States was $13.8 \%$ at 24 months. $^{18}$ The cumulative risk of epilepsy among 2826 persons with TBI in China was 5.0\% after 3-year follow-up. ${ }^{19}$ The 30 -year cumulative incidence was $2.1 \%$ for mild TBI, $4.2 \%$ for moderate TBI, and $16.7 \%$ for severe TBI in a cohort from the United States. $^{8}$ The cumulative probability of epilepsy among 137 persons with TBI in Italy was $10 \%$ at 6 months and $17 \%$ at 12 months. $^{20}$ The cumulative incidence of epilepsy among 3,093 TBI patients in China was 9.8\% at 24-month follow-up. ${ }^{21}$ Among 275 patients in England followed for a minimum of 4 years, 28 persons (10\%) developed epilepsy. ${ }^{22}$ The absolute risk may be even higher in institutionalized persons $(25 \%$ after 12-year follow-up) ${ }^{23}$ and combat brain injury (42\% after 30-35-year follow-up). ${ }^{24}$

Although different in a brain injury population, disease stratification and follow up, these studies indicate that the risk of epilepsy is very high the first years after diagnosis, and that the cumulative incidence continues to increase with length of follow-up. To study this in further detail, we used data from the study by Annegers, ${ }^{8}$ and calculated the cumulative probability of unprovoked seizures following brain injury by severity. We estimated the risk of epilepsy year $<1$, year 1 to 4 , year 5 to 9 , and year 10 to 30 after brain injury by dividing the number of new-onset seizures in the time interval with the number of persons with brain injury alive without epilepsy at the start of follow up of the individual time intervals. ${ }^{8}$ - Fig. 5 shows their cumulative risk of epilepsy. However, although the cumulative probability of epilepsy continues to increase, the yearly probability of posttraumatic epilepsy decreases sharply the first years after brain injury. We calculated the average yearly 


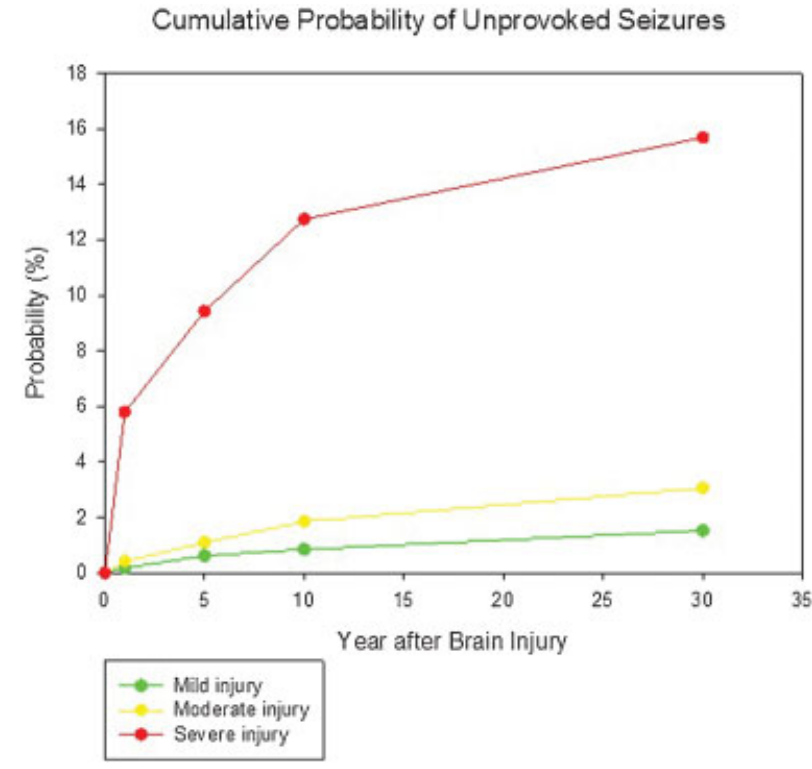

Fig. 5 Cumulative probability of unprovoked seizure, adapted from Annegers JF et al. ${ }^{8}$

absolute risk of epilepsy by dividing the number of new-onset epilepsy with the number of persons at risk at the start of the time interval divided by the number for years in the time interval (-Fig. 6). Although the absolute risk of posttraumatic epilepsy is high the first year after diagnosis-especially following severe brain injury-this absolute risk decreases sharply and by 5 years of follow-up the yearly absolute risk is below $1 \%$ for all types of brain injury including severe brain injury (-Fig. 6).

\section{Treatment and Prevention of Epilepsy following Traumatic Brain Injury}

As with other epilepsies with focal onset, posttraumatic epilepsy can be notoriously difficult to treat, and seizure

Yearly absolute probability of unprovoked seizures after brain injury

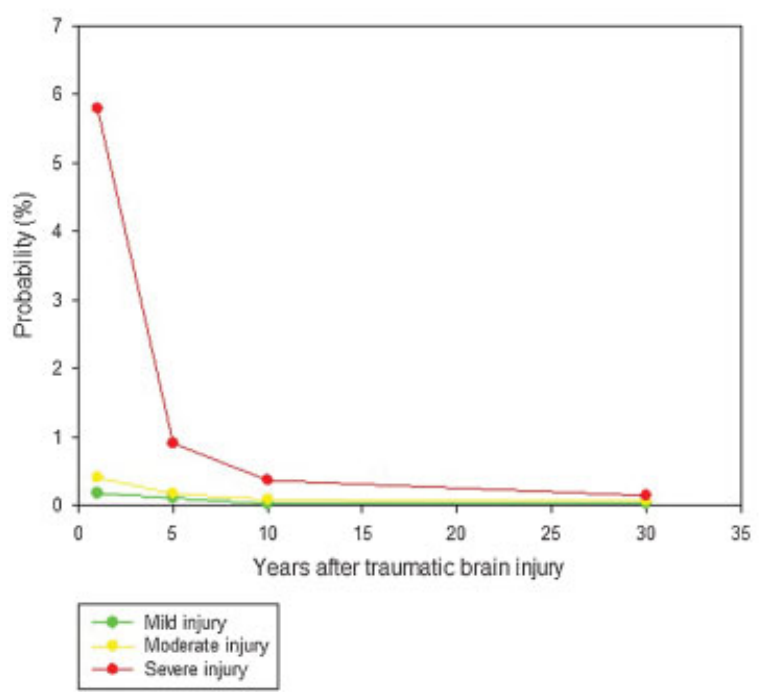

Fig. 6 Yearly absolute probability of unprovoked seizures after brain injury, adapted from Annegers JF et al. ${ }^{8}$

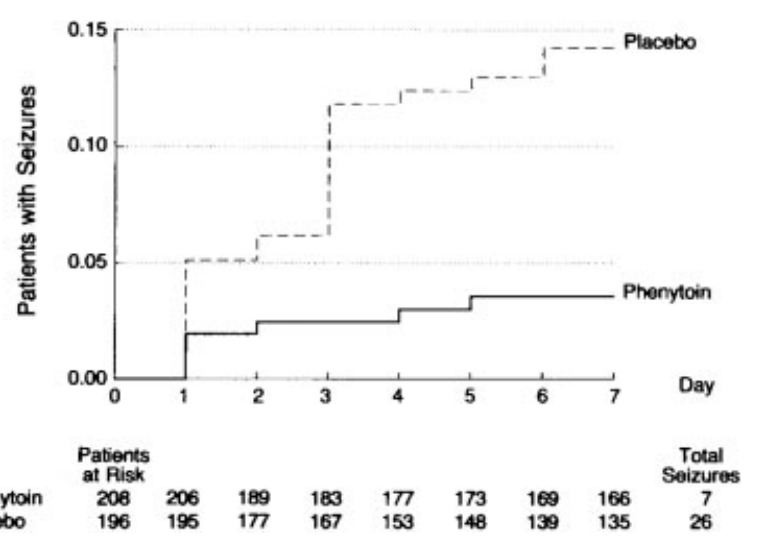

Fig. 7 Cumulative fraction of patients with early seizures. ${ }^{9}$

freedom may be hard to obtain. ${ }^{25}$ However, epilepsy after TBI is potentially preventable because posttraumatic seizures may follow the injury only after several years ( - Fig. 4). 8,14,15,26,27 This provides an "open treatment window," and there have been several clinical trials of antiepileptic drugs to try to prevent posttraumatic epilepsy. In addition, development of posttraumatic epilepsy has been suggested, in part, to rely on activation of the N-methyl-Daspartate- (NMDA-) glutamate receptors, an effect that is blocked by extracellular $\mathrm{Mg}^{2+}$ ions. Magnesium has therefore also been studied in patients with TBI in an effort to prevent epileptogenesis; the neuroprotective properties of magnesium are believed to be mediated by their effect on NMDA receptors ${ }^{9,26,28-30}$

Three major trials stand out as conclusive for the effects of antiepileptic drugs in the prevention of posttraumatic epilepsy. ${ }^{9,28-30}$ In these trials, sodium valproate, carbamazepine, and phenytoin did not prevent the development of late seizures (epilepsy) after moderate-to-severe TBI. However, phenytoin treatment prevented early seizures in the first week after TBI ( - Fig. 7). There were similar results of a protective effect with carbamazepine against early posttraumatic seizures, but carbamazepine also failed to protect against late seizures (epilepsy). ${ }^{30}$ Magnesium did not provide protection against posttraumatic epilepsy in a human clinical trial. $^{31}$

\section{Conclusion}

Traumatic brain injury carries an increased risk of epilepsy that correlates with the severity of the brain injury. Posttraumatic epilepsy accounts for less than $10 \%$ of epilepsy, but constitutes one of only a few potentially preventable causes of epilepsy. However, despite several well-controlled human studies, there is no current preventive treatment available in humans. Primary prevention is therefore the only proven way to prevent posttraumatic epilepsy. In that respect, it is encouraging that the hospitalization rate for TBI has fallen among the civilian population, but it is not clear whether this reflects a change in admission practice or a true decrease in TBI incidence. The decrease among the civilian population 
may be counterbalanced by a concerning increased incidence of hospitalization for TBI among the military population. ${ }^{32}$

\section{References}

1 Pickelsimer EE, Selassie AW, Gu JK, Langlois JA. A population-based outcomes study of persons hospitalized with traumatic brain injury: operations of the South Carolina Traumatic Brain Injury Follow-up Registry. J Head Trauma Rehabil 2006;21(6):491-504

2 Centers for Disease Control and Prevention. Traumatic Brain Injury in the United States: Emergency Department Visits, Hospitalizations, and Deaths, 2002-2006. Atlanta, GA: Centers for Disease Control and Prevention; 2012

3 Olesen J, Gustavsson A, Svensson M, Wittchen HU, Jönsson B; CDBE2010 study group; European Brain Council. The economic cost of brain disorders in Europe. Eur J Neurol 2012;19(1): 155-162

4 Hesdorffer DC, Benn EK, Cascino GD, Hauser WA. Is a first acute symptomatic seizure epilepsy? Mortality and risk for recurrent seizure. Epilepsia 2009;50(5):1102-1108

5 Lowenstein DH. Epilepsy after head injury: an overview. Epilepsia 2009;50(2, Suppl 2):4-9

6 Beghi E, Carpio A, Forsgren L, et al. Recommendation for a definition of acute symptomatic seizure. Epilepsia 2010;51(4): 671-675

7 Thurman DJ, Beghi E, Begley CE, et al; ILAE Commission on Epidemiology. Standards for epidemiologic studies and surveillance of epilepsy. Epilepsia 2011;52(7, Suppl 7):2-26

8 Annegers JF, Hauser WA, Coan SP, Rocca WA. A population-based study of seizures after traumatic brain injuries. N Engl J Med 1998; 338(1):20-24

9 Temkin NR, Dikmen SS, Wilensky AJ, Keihm J, Chabal S, Winn HR. A randomized, double-blind study of phenytoin for the prevention of post-traumatic seizures. N Engl J Med 1990;323(8):497-502

10 Temkin NR. Risk factors for posttraumatic seizures in adults. Epilepsia 2003;44(10, Suppl 10):18-20

11 Fisher RS, Acevedo C, Arzimanoglou A, et al. ILAE official report: a practical clinical definition of epilepsy. Epilepsia 2014;55(4): 475-482

12 Fisher RS, van Emde Boas W, Blume W, et al. Epileptic seizures and epilepsy: definitions proposed by the International League Against Epilepsy (ILAE) and the International Bureau for Epilepsy (IBE). Epilepsia 2005;46(4):470-472

13 Haltiner AM, Temkin NR, Dikmen SS. Risk of seizure recurrence after the first late posttraumatic seizure. Arch Phys Med Rehabil 1997;78(8):835-840

14 Christensen J, Pedersen MG, Pedersen CB, Sidenius P, Olsen J, Vestergaard M. Long-term risk of epilepsy after traumatic brain injury in children and young adults: a population-based cohort study. Lancet 2009;373(9669):1105-1110

15 Ferguson PL, Smith GM, Wannamaker BB, Thurman DJ, Pickelsimer EE, Selassie AW. A population-based study of risk of epilepsy after hospitalization for traumatic brain injury. Epilepsia 2010;51(5): 891-898

16 Emanuelson I, Uvebrant P. Occurrence of epilepsy during the first 10 years after traumatic brain injury acquired in childhood up to the age of 18 years in the south western Swedish population-based series. Brain Inj 2009;23(7):612-616

17 McGuire SA, Marsh RW, Sowin TW, Robinson AY. Aeromedical decision making and seizure risk after traumatic brain injury: longitudinal outcome. Aviat Space Environ Med 2012;83(2):140-143

18 Englander J, Bushnik T, Duong TT, et al. Analyzing risk factors for late posttraumatic seizures: a prospective, multicenter investigation. Arch Phys Med Rehabil 2003;84(3):365-373

19 Zhao Y, Wu H, Wang X, Li J, Zhang S. Clinical epidemiology of posttraumatic epilepsy in a group of Chinese patients. Seizure 2012;21(5):322-326

20 Angeleri F, Majkowski J, Cacchiò G, et al. Posttraumatic epilepsy risk factors: one-year prospective study after head injury. Epilepsia 1999;40(9):1222-1230

21 Wang H, Xin T, Sun X, et al. Post-traumatic seizures-a prospective, multicenter, large case study after head injury in China. Epilepsy Res 2013;107(3):272-278

22 Jennett WB, Lewin W. Traumatic epilepsy after closed head injuries. J Neurol Neurosurg Psychiatry 1960;23:295-301

23 Asikainen I, Kaste M, Sarna S. Early and late posttraumatic seizures in traumatic brain injury rehabilitation patients: brain injury factors causing late seizures and influence of seizures on longterm outcome. Epilepsia 1999;40(5):584-589

24 Raymont V, Salazar AM, Lipsky R, Goldman D, Tasick G, Grafman J. Correlates of posttraumatic epilepsy 35 years following combat brain injury. Neurology 2010;75(3):224-229

25 Hakimian S, Kershenovich A, Miller JW, et al. Long-term outcome of extratemporal resection in posttraumatic epilepsy. Neurosurg Focus 2012;32(3):E10

26 Christensen J. Traumatic brain injury: risks of epilepsy and implications for medicolegal assessment. Epilepsia 2012; 53(4, Suppl 4):43-47

27 Schmidt D, Friedman D, Dichter MA. Anti-epileptogenic clinical trial designs in epilepsy: issues and options. Neurotherapeutics 2014;11(2):401-411

28 Temkin NR. Antiepileptogenesis and seizure prevention trials with antiepileptic drugs: meta-analysis of controlled trials. Epilepsia 2001;42(4):515-524

29 Temkin NR. Preventing and treating posttraumatic seizures: the human experience. Epilepsia 2009;50(2, Suppl 2):10-13

30 Glötzner FL, Haubitz I, Miltner F, Kapp G, Pflughaupt KW. [Seizure prevention using carbamazepine following severe brain injuries]. Neurochirurgia (Stuttg) 1983;26(3):66-79

31 Temkin NR, Anderson GD, Winn HR, et al. Magnesium sulfate for neuroprotection after traumatic brain injury: a randomised controlled trial. Lancet Neurol 2007;6(1):29-38

32 Chen LL, Baca CB, Choe J, Chen JW, Ayad ME, Cheng EM. Posttraumatic epilepsy in Operation Enduring Freedom/Operation Iraqi Freedom veterans. Mil Med 2014;179(5):492-496 